\title{
Building the Business Case for Wireless Sensors in a Factory Setting
}

\author{
Koen Casier, Lieven Tytgat, Sofie Verbrugge, Mario Pickavet, Ingrid Moerman \\ Ghent University, IBBT - Gaston Crommenlaan 8, bus 201 \\ 9050 Ghent (Belgium) \\ Koen.casier@intec.ugent.be
}

\begin{abstract}
With the advent of the low complexity wireless technology Zigbee, different cheaper and more energy efficient wireless transceivers became available on the market. These chipsets are gradually introduced in different small scale wireless applications. Especially in cases of wireless sensor networks, the low energy consumption and cost efficiency are a real gain. As such it is often used in small non-critical monitoring type battery powered sensor installations. In more time-critical applications, all wireless signals should be better orchestrated to avoid collisions, especially with Wi-Fi. So called spectral sensing engines are able to scan the wireless spectrum and severely reduce the probability of collisions, even between different technologies as in the Wi-Fi $\Leftrightarrow$ Zigbee case. This paper investigates if both technologies - with or without spectral sensing engine - could lead to more cost efficient installations in more critical sensor network installations, where typically today a wired solution is used. As a realistic benchmarking case, we consider an installation in a factory in which many different types of sensors - critical and background - are scattered over the production facility and are used in controlling all processes. In this paper we constructed cost models for the wired and both wireless solutions. The results clearly show that wireless sensors could lead for smaller installations to large cost reductions (up to $50 \%$ ) and give a more detailed view on the tradeoffs in a wired vs. wireless sensor network installations. This model can be used for identifying the most cost efficient sensor network for different factory installations, as well as beyond the realm of factories. The economic model is more generally applicable for sensor networks spread over large areas including mission critical and non-critical sensors.
\end{abstract}

Keywords- sensor network; Zigbee; Wi-Fi; sensing engine; wireless automation; techno-economic; business case;

\section{INTRODUCTION}

Factory production units are currently highly automated production lines which contain lots of sensors to guarantee the well functioning of the processes. During the installation and maintenance of such a production unit, many tasks involve the placement, upgrade or replacement of sensors to control the unit. Clearly radio sensors could greatly decrease the installation effort and as such reduce the total cost of the factory installation and maintenance (see Figure 1).

Current radio technologies allow to provide the required bandwidth to connect wireless sensors in a cost efficient way. Zigbee promises to offer the lowest price components and could as such be easily built into the sensor at virtually no extra cost. However wireless devices are currently very well adopted and the many different technologies and devices share the same spectrum. These different technologies are usually not designed to be compatible, and therefore they tend to jam each other and block the optimal functioning of the network. If the channels of the devices are disrupted too much, this might lead to a total collapse of the network, in the worst case resulting in discontinuation of the production because of the blocking of the important sensor traffic. This situation is not tolerable in a factory setting and as such there is little to no industrial introduction of wireless sensory devices.

This problem can be mitigated by adding a crosstechnology spectral sensing engine, a new evolution in wireless hardware, to the wireless sensor devices. Such a crosstechnology spectral sensing engine is capable of listening to the wireless channels of various wireless technologies, and interpreting when the channel is free for transmission. Using the right techniques and protocols learned from the cognitive radio field of research [1], these sensing engines can effectively reduce cross-technology collisions and increase the wireless communication reliability of the sensors significantly. This increased level of reliability allows an opportunity for cost reductions in the installations, maintenance and upgrades of the sensors albeit at the cost of additional sensing engines.

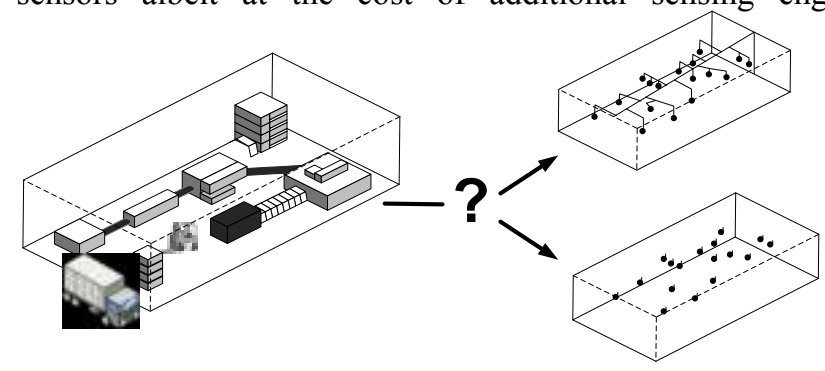

Figure 1: Wired versus wireless factory automation

Clearly it is important to know when wireless sensors could be more cost-efficient in a factory setting. Therefore we build a quantitative cost estimation model in the first part of this paper, (Figure 2). This model consists of four building blocks. In the first block, we delve into the details of the installation and quantify the costs, which allow us to make a detailed and well balanced comparison of different options in sensor installation. In this quantification, we distinguish between the different types and requirements for the sensors and the locations of installation. In the second block we complement this sensor installation cost model with an energy model for the wireless sensor nodes, allowing dimensioning of the batteries, estimating their replacement time, and adding both as extra cost factors to the cost model. In the third block we build an 
operational cost model estimating all costs for maintenance and replacement of sensor nodes or batteries and of upgrades to the sensor network. Finally, in the fourth block, we construct a reliability model for the sensor nodes which is used to determine the errors occurring due to communication faults, and their resulting costs. These four building blocks are implemented for fixed and both wireless (with or without sensing engines) sensor networks and give as such a fully comparative model for the different installation possibilities for a generic sensor network installation in a factory setting based on the full cost and performance model.

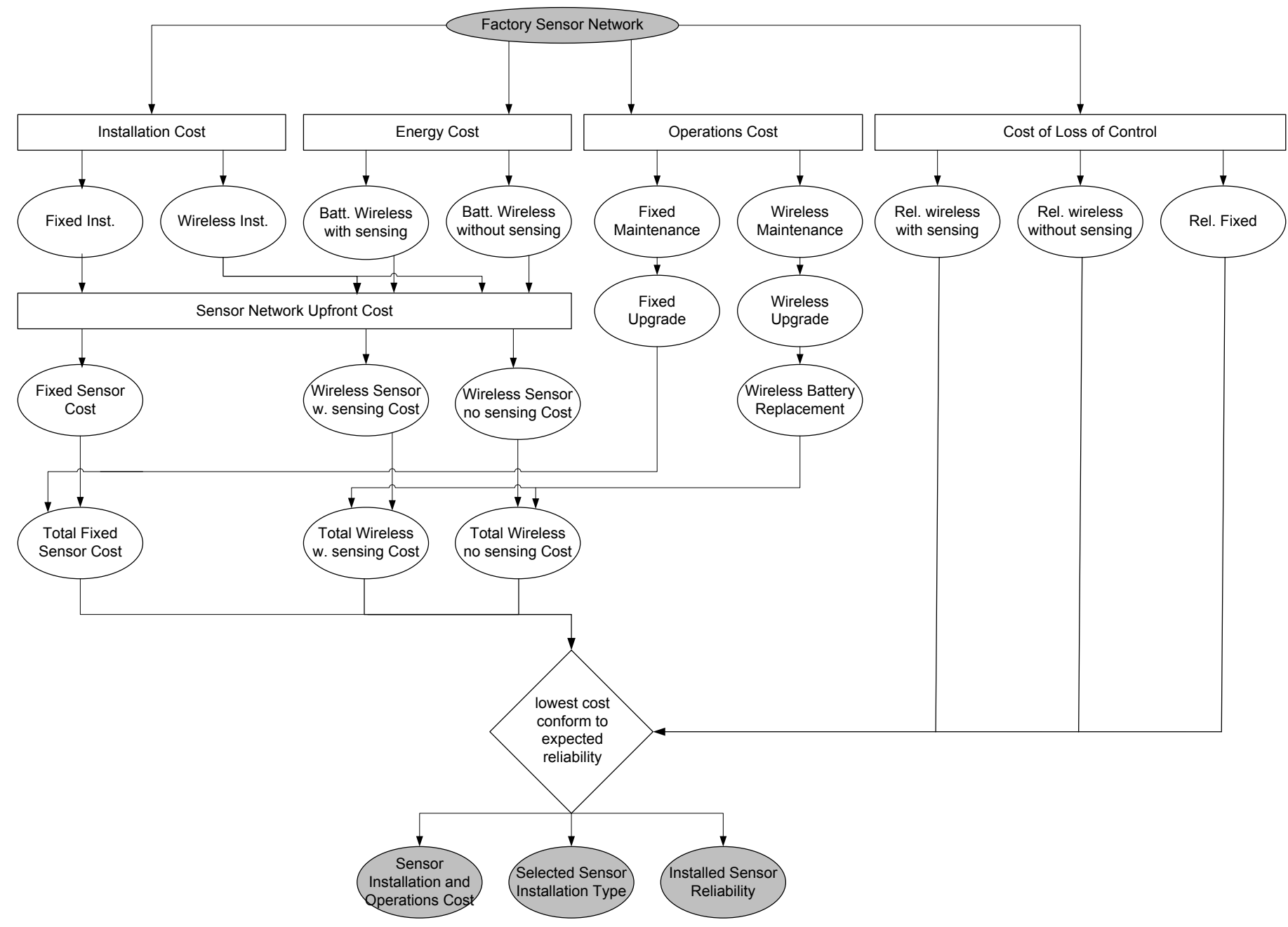

Figure 2: Structured cost model for a wired and wireless with and without sensing engine factory sensor network installation

In the second part of this paper the model is applied to realistic factory settings differing in size of the factory, setup of the production facility, number and placement of sensors, information on reliability and maintenance periods, etc. It shows the most important parameters in the costs of installation and maintenance of a sensor network in a factory for the wired and both wireless cases.

\section{WHAT'S IN A FACTORY}

Every factory basically consists of a giant concrete box possibly split in different production areas in each of which machinery is placed in a logical chain. Generally, there are highly automated production clusters separated by clusters of lower automation. In the highly automated production clusters, there is a large need for many types of sensory devices connected to controllers. Sensory devices can be integrated or attached to the machinery, coupled to the chain, overseeing a production unit, linked to the product, etc. Sensors can also differ in the physical variable they measure and in their sensitivity, maximum deviations, drift, etc.

We consider three cost classes of sensory devices; low complexity and low cost sensors are estimated at an average cost of $€ 15$, mid range sensors are set at $€ 25$ and high cost complex sensors have an associated cost of $€ 75$. All prices are based on a sample of different available sensors on the market. 
For the setup of the factory it is also important to indicate the refresh rate of the sensors. We distinguished three classes; background sensors which serve as non-critical measurements and have a refresh rate around once per minute (or less), mid rate sensors with a refresh rate of once per 5 seconds and finally the critical sensors which refresh their measurements every half a second. Data of one measurement consists of 3 bytes and 4 measurements are bundled together per refresh.

The following two subsections show how to calculate the costs of a sensor network installation either in a wired solution or in a wireless solution. The models for both consist of the building blocks from the structure shown in Figure 2. This model is completed with a cost estimation of any failure requiring a shutdown of (part of) the factory. Everything combined gives the cost model which will be used in the next section.

\section{A. Wiring it all up}

Current practices for sensor installations use one of three connection types. A first way to connect all sensors is through a single wire per sensor. A second way is achieved by using so called multicables. Multicables consist of a number of wires (eg. 8), and as such using 2 of them for powering, the remainder can be used for coupling sensors in exactly the same manner as traditional sensors allowing for 6 sensors next to each other. Finally a standardized medium (eg. Ethernet, PLC bus, etc.) is considered as their underlying bus infrastructure. As such there are mainly three different topologies, which still allow mingling:

\section{A system with one sensory device per wire}

2. Multicable connected sensory devices on one of the free wires.

3. A digital system in which all devices are coupled to a bus. (Ethernet, PLC bus, etc.) This requires more complex digital circuitry and microcontroller software per sensory device.

We will assume that all devices are either connected through a multicable system, or through a bus system. The total cost of a wired sensor network consists of three parts which we will elaborate on in the following parts.

\section{Cables for wiring all sensors}

The length in cabling is calculated by means of an analytical model in which all sensors have been scattered around the center of the cluster. For small clusters, we estimate the different sensors to be equally spaced in the cluster at $2 / 3$ th off radius. Cable length for larger clusters is calculated by means of an analytical model in which the sensors are equally spaced in a square with side equal to the radius given for the cluster. The cost for a cable amounts $€ 1.15 / \mathrm{m}$ in line with the current market cost of Cat5E Ethernet cable.

a. Cabling all sensors to the cluster center In the center of each cluster, we consider an aggregation hub able to aggregate digitally the traffic coming from 8 ports each serving up to 6 parallel devices as proposed in the sensor configuration 2 before. The cost of one such hub is 120 euro consisting of the cost of a standard Ethernet hub (20 euro) and a dedicated microcontroller for controlling and aggregating the sensor traffic attached to this (100 euro)

b. Connecting all clusters to one central control point All clusters are to be connected to one central point and we assume all clusters to be equally spaced over the area with $50 \mathrm{~m}$ in between the clusters.

\section{Planning and installation of all cabling}

Connecting all sensors involves some planning where to put the cables and the installation of all cables. It is estimated that $1 \mathrm{~m}$ of cable will require some way of fixing the cable on the ceiling, an operation taking on average 6 minutes per meter fixating.

\section{Maintenance of all cables}

In factories, cables used by running machinery can be broken by any passing machines, restructuring, wear, etc. While there is only a very small chance for a cable rupture per meter, large installations could be troubled with such event sooner or later, and any downtime in sensors could well lead to considerable losses in the production of the factory. The costs of downtime will be very depending on the type of sensor device, the rate at which it should communicate and the types of processes running or goods produced in the factory. For each case of cable break, we expect the easiest and fastest repair actions are to replace the failing cable and reattach both sensors between which this cable is attached. We estimate the chance of cable break to be equal to 1 per $20 \mathrm{~km}$ per year.

\section{B. Wireless $=$ just plug-in?}

With the introduction of the lower complexity Zigbee wireless protocol, much cheaper and energy efficient wireless transceivers are available on the market. Wireless sensors will hardly increase in size and can be much easier installed in a networked solution. Considering a production hall, typically the distances are not problematic for wireless transport. Zigbee can provide a bandwidth of $250 \mathrm{kbps}$ and, as mentioned before the data of four measurements is combined within one packet. Including the header the packet sent out has a size of 125 bytes which takes $4 \mathrm{~ms}$ to send out. Taking into account an additional back-off period randomly with average of $1.1 \mathrm{~ms}, 0.6 \mathrm{~ms}$ for RTS $\backslash$ CTS (requestlclear to send) and $0.6 \mathrm{~ms}$ for ACKs (acknowledge receipt), the total transmission takes somewhere around $7 \mathrm{~ms}$. This allows up to 68 critical sensor devices to use the same channel when perfectly scheduled. Mid range sensors are limited to 860 devices and up to 8000 background sensors can be working together.

The cost structure for a wireless sensor network is significantly different as the network is self configuring and the sensors can simply be attached to the location where they should perform the measurements. The cost structure of the wireless solution is as such fairly straightforward as only the wireless transceiver part will form an extra cost which for a Zigbee module will be a cost of 20 euro per sensor. For each sensor installed such a module should be incorporated. 
Just as in the case of the wired sensors, wireless sensors can also lead to failures due to malfunctioning of the medium. Clearly, cables cannot be broken. On the other hand, when multiple wireless sensors try to send out their measurement data to the central controller at the same time, they will interfere each other's signal which will lead to a short failure period. The main system for dealing with such failures is a retransmit after a random back-off period for the considered data. This will take some time and could lead to missing the opportunity to effectively control the process and a failure scenario (either proactive safe failure or hard failure with forced stop of the processes). This will happen especially when WiFi or other wireless technologies are co-located. To account for this case, either the wireless sensor nodes should use WiFi instead of Zigbee which allows them to have less interference from other signals, or all devices (e.g. Wi-Fi and Zigbee) could have a built-in spectral sensing device, capable of sensing the medium to check whether it is free of any kind of wireless signal and can be used for sending out its measurements. In combination with scheduling software and priorities this leads to an optimal usage of the wireless medium. The first approach, switching to WiFi will lead to an increase of the cost to somewhere around 40 euro, comparable to the increase in cost which would be incurred when incorporating a sensing engine (somewhere around 20 euro per piece). As the second approach - adding a sensing engine - will lead to lower power consumption for the wireless sensor and a higher availability, this approach is used in the remaining calculations.

Availability of the wireless network is defined in terms of chance of missing a measurement update which might render the process unstable or under-controlled and which might lead to failure, breakage to machines, etc. Depending on the process and the amount of outdated measurements this can lead to a temporary shutdown of the factory or part of the factory. In the situation in which a sensing engine is used, the chances of collisions with a wireless signal are small and no retransmissions will be required.

Finally all wireless sensor devices will require batteries to feed their functioning. We base the costs for battery on bulk sale such as [2] which minimally leads to a fitted cost function per Watt-hour (Wh) equal to $0,2018 \times$ Quantity $^{-0,149}$ $\left(\mathrm{R}^{2}=0,9974\right)$ and maximally leads to a fitted cost function per Wh equal to $0,2154 \times$ Quantity $^{-0,085}\left(\mathrm{R}^{2}=0,9755\right)$. In this the quantity refers to the amount of batteries to buy at once. We consider generic AA batteries with an energy content of $3 \mathrm{Wh}$ per battery. As such the cost per battery for a given quantity can be calculated by multiplying the outcome of this function with 3 (Wh/battery). Using the power dissipation values for all states of a Zigbee transceiver and microcontroller unit (MCU) as given in Table I, we can calculate the power dissipation for the three types of refresh rates. For a refresh rate of $0.5 \mathrm{~s}$ (critical sensors) this is equal to $0,8 \mathrm{~mW}$, for a refresh rate of $5 \mathrm{~s}$ (mid range sensors) this is equal to $0,22 \mathrm{~mW}$ and finally for a refresh rate of $60 \mathrm{~s}$ (background sensors) this is equal to $0,16 \mathrm{~mW}$. In the base case we aimed for all sensors to be equal in form factor and a minimal estimated life time for the critical sensors of 1 year based on AA batteries. For this 3 batteries are required leading to a minimum estimated lifetime of $1.1 \mathrm{y}$ for the critical sensors, $2.3 \mathrm{y}$ for the mid range sensors and $2.6 \mathrm{y}$ for the background sensors. Based on nominal power dissipation this is even an estimated lifetime of $2 \mathrm{y}, 8 \mathrm{y}$ and $11 \mathrm{y}$ respectively for the sensors.

TABLE I: ZIGBEE TRANSCEIVER POWER DISSIPATION PARAMETERS

\begin{tabular}{|lrr|l|}
\hline specs & Nom & \multicolumn{3}{c|}{ Max } \\
\hline Supply & 2,85 & 3,6 & $\mathrm{~V}$ \\
\hline RX (receive) & 21,8 & 23 & $\mathrm{~mA}$ \\
\hline TX (transmit) & 19,5 & 21 & $\mathrm{~mA}$ \\
\hline MCU on/radio off & 1,8 & 2,4 & $\mathrm{~mA}$ \\
\hline MCU idle & 0,0545 & 1,2 & $\mathrm{~mA}$ \\
\hline MCU standby & 0,0051 & 0,021 & $\mathrm{~mA}$ \\
\hline
\end{tabular}

\section{Costs of losing control}

Losing contact with the sensors can lead to situations in which it is no longer safe to keep the production facility running, as dangerous situations for machines (e.g. breakage), people or production (faulty products) could emerge. In case of late or missed measurements the standard action could well be to automatically go into a safe shutdown of the production in order to avoid high losses, machine damage or even danger. However, such a safe shutdown scenario in itself can lead to considerable losses in working time, production capacity, startup costs, scrap created, etc.

The costs of losing control and performing a safe shutdown and the limits on the sensors measurement timings will be very dependent on the specifics of the factory such as the type of process, product, organization of the factory, machinery, etc. As a base case we consider a fast, but non-costly shutdown process, which will only cost 100 euro per hour of shutdown. However a shutdown will occur after not receiving any of the sensor messages within the allowable delay depicted before (100ms, 1s and $12 \mathrm{~s}$ for critical, mid rate and background sensors respectively).

This cost of losing control is applicable for every solution and is caused by the following factors:

\section{Failing sensor}

Sensor failure rate is $2 \%$ per year which corresponds to a maximum lifetime of 50 years and a mean time between failures of 25 years.

2. Cable breakage, while this might affect many sensors at once, we only considered this to have the same effect as an out of time measurement for one sensor. Still the repair time for the cabling is larger than the out of time of a wireless failure.

A cable break is assumed to take place once per year per $20 \mathrm{~km}$ cable. We expected this to be 10 times higher than in case of an access network [3] as the cables are not protected by burying and ducting, but are openly attached to the ceiling and/or machinery. 
3. Out of time measurement due to wireless interference.

Wireless interference occurs when two devices send out their signal at the same time and result in packets being lost. In a straightforward collision detecting protocol, this will often be the case when many sensors are placed in the same area. Within the sensor network, this can be avoided by using some slotted approach in which the different sensor devices get a slot assigned and only send out their data in this time-slot. In this manner all collisions between Zigbee devices can be avoided on condition that there are no more slots required than available. As mentioned before the maximum allowable amount of sensors in this case is 68,860 and 8000 respectively critical, mid rate and background.

However, when regular $\mathrm{Wi}-\mathrm{Fi}$ is co-located with the sensor network, a lot of collisions will unavoidably occur resulting in a lot of retransmission. This process takes considerable more time and as such leads to even more collisions and eventually a collapse of the sensor network, failing to deliver any sensor measurements. We used the mathematical model presented in [4] and [5] to calculate the probabilities of having a successful transmission within the allowable delays for each sensor type. Given the allowable delays and the WiFi intensity, it seems that with only $10 \% \mathrm{WiFi}$ intensity (eg. $0.1 \mathrm{Mbps}$ for $1 \mathrm{Mbps} \mathrm{Wi}-\mathrm{Fi}$, $5.4 \mathrm{Mbps}$ (indicated on Figure 3) for $54 \mathrm{Mbps} \mathrm{Wi}-\mathrm{Fi}$ ), the non-sensing slotted Zigbee solution leads to a multitude of failures, as is also shown in Figure 3 based on this same publication.

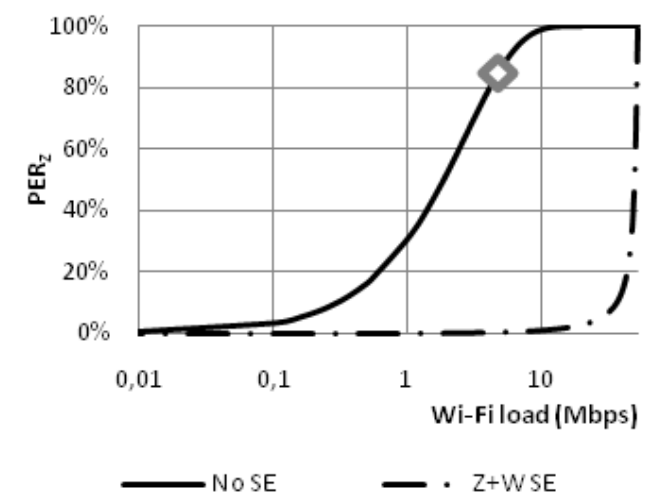

Figure 3: Influence of WiFi intensity (with indication of $10 \%$ ) on the packet error rate (PER) for Zigbee without vs. with sensing.

Using the sensing engines, all devices can detect all transmissions - whether Wi-Fi or Zigbee - and thus avoid most collisions. The solution using sensing engines can as such guarantee a reliable transmission as long as the total amount of sensor traffic is not higher than the maximal sensor throughput multiplied by the percentage of the time in which there is no Wi-Fi traffic.

\section{TRADEOFFS IN REALISTIC FACTORY SETTINGS}

The cost model constructed in the previous section is generic for all types of factory settings. Based on this cost model, we take a closer look into the tradeoffs between the different alternatives - wired, wireless without sensing and wireless with sensing. The most important impact will of course come from the amount, type and placement of sensors which is tackled first. Next to that also the impact of changes in the costs of the different components are considered.

\section{A. Amount, type and placement of sensors}

For a small amount of sensors, clearly a wireless solution will be more cost efficient, while for increasing sensor count a wired solution will become more and more cost efficient.

Figure 4 gives a view on the relative costs of installation and maintenance of a wired and of wireless sensor network with and without sensing for an increasing amount of sensors. For each of the three predefined refresh rates all relative costs are expressed in percentage investments on top of the most cost-efficient solution. Wireless solutions with or without sensing are in all cases more cost efficient for up to 10 sensors in one cluster (e.g. machine). This is even much higher in case of less critical sensors and without sensing engines (up to 36 sensors). Even beyond this point the cost of a wireless sensor network is only $20-30 \%$ higher than for a wired solution.

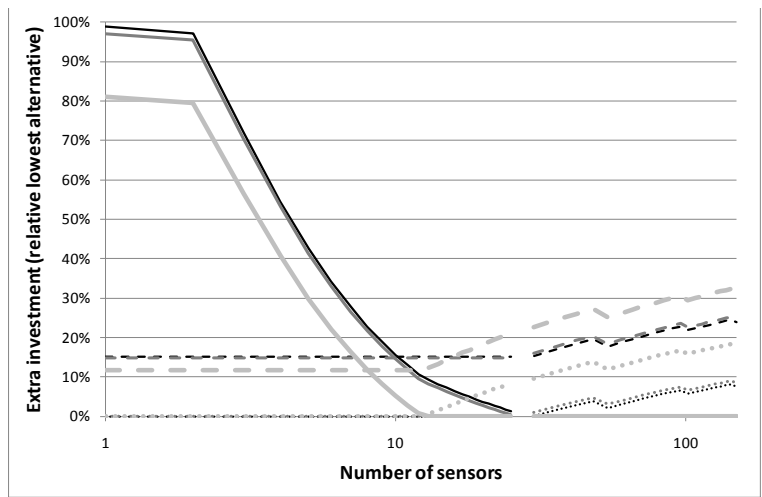

Figure 4: Relative higher investments for fixed (solid) vs. wireless with (striped) and without (dotted) sensing engines in terms of number of sensors. Also indicated are background (black) vs. low rate (grey) and critical (light grey) sensor refresh rates.

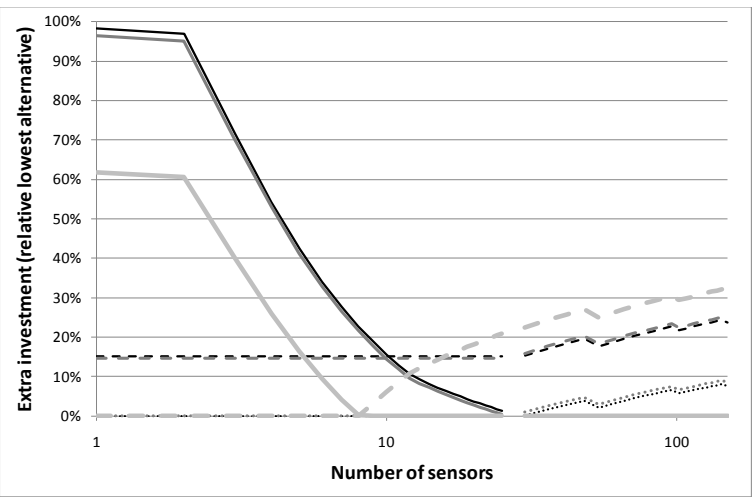

Figure 5: Relative higher investments in terms of number of sensors taking into account the costs for loss of control

Figure 5 gives a view on the costs taking into account the costs occurred due to loss of control. Clearly especially the critical sensors are better installed with a wired solution, even as soon as there are 8 sensors installed. Critical sensors can never be installed without sensing engines in case $\mathrm{WiFi}$ 
background intensity is at $10 \%$. This clearly indicates the gain in using sensing engines as an extension to Zigbee transceivers.

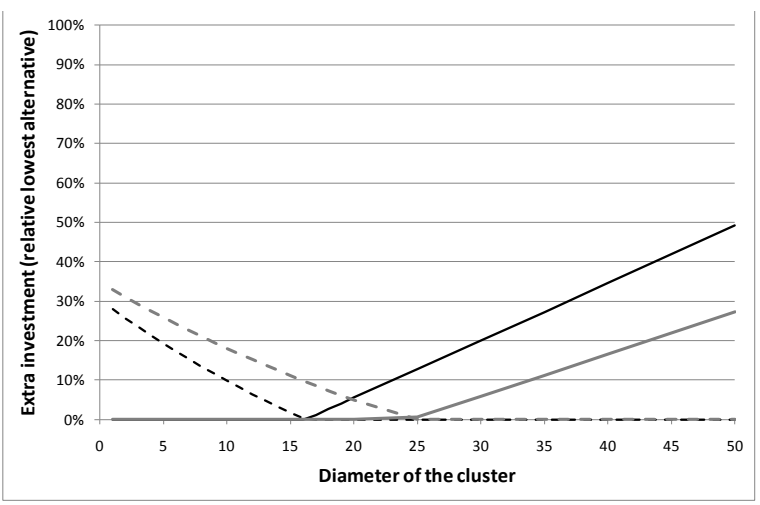

Figure 6: Relative higher investments for fixed (solid) and wireless with sensing engine (striped) in terms of diameter of the cluster and amount of sensors 15 (black) or 30 (grey)

Figure 6 shows how for larger clusters, the wiring cost leads to a higher cost with a typical switching point at 15 to 25 meter respectively for 15 to 30 sensors. For very large (factory) buildings with a small amount of evenly spread sensors, the costs can be up to $50 \%$ higher for a wired solution than for a wireless solution.

\section{B. Cost Parameters}

With an increase in production and maturing of technology, prices typically drop and integration makes them even drop more. In the case of the sensing engine this could provide an important gain for usage in time critical applications.

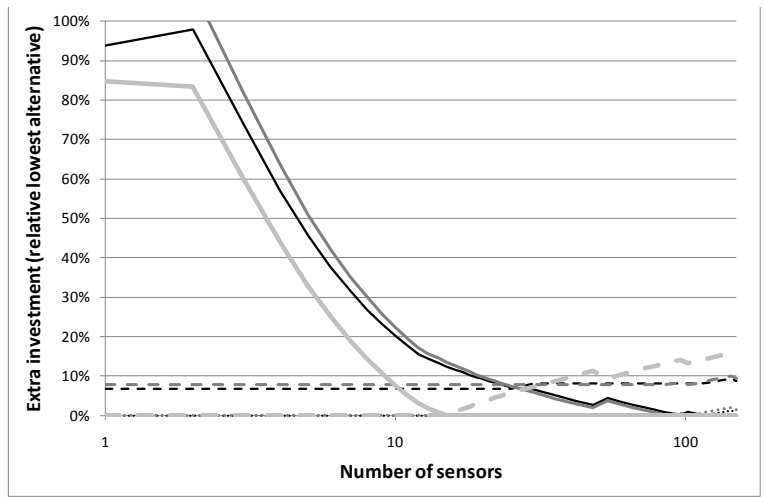

Figure 7: Relative higher investments in terms of number of sensors for a lower wireless transceiver and sensing engine cost

In Figure 7 the relative extra investments over the most cost efficient solution are shown for the three alternatives when the Zigbee transceiver drops in price to 10 euro and the sensing engine drops to the same price. It is not unreasonable to expect an even higher price drop especially if the sensing engine would at one point in time be integrated into the Zigbee transceiver. Clearly a much higher amount of sensors can be installed more cost efficiently using wireless technologies. Up to 100 background sensors and over 20 critical sensors can be accommodated more cost efficiently using wireless, in the latter case wireless with sensing engine. Additionally the extra investments for a network with 150 sensors will only be $15 \%$ more than in case of a wired sensor network.

\section{CONCLUSIONS}

Factories have highly automated production lines in which many sensory devices are controlling the processes performed. The installation of such sensors is not always straightforward, and can be a very costly process. In this field the low cost wireless technology Zigbee could be a promising solution. When equipped with a wireless transceiver and a battery pack, installing or replacing sensors is only a question of attaching the sensor at the right location. Still the Zigbee technology is to sensitive to interference from $\mathrm{WiFi}$, leading to an unreliable situation. The novel development of a spectral sensing engine allows, when coupled with a transceiver, to scan the spectrum and avoid any collisions whenever possible.

In this paper we compared the three different solutions wired, Zigbee without sensing and Zigbee with sensing considering their economic impact on installation, powering, maintenance and reliability. The results indicate that with current pricing of Zigbee module a cost efficient solution can be provided for up to 30 sensors in case they are non-critical without a sensing engine. Only up to 10 critical sensors can be accommodated when using a sensing engine, but this situation quickly changes drastically when a decreasing price evolution is taken into account allowing up to 100 non-critical sensors and over 20 critical sensors in one location.

The model developed in this paper opens the search for different other cases in which critical sensors without wiring will lead to important savings and are not in use today. In this estimating the cost of installation, maintenance, powering and upgrading a reliable wireless sensor network will remain very important

\section{REFERENCES}

[1] Pollin, S.; Tan, I.; Hodge, B.; Chun, C.; Bahai, A.; , "Harmful Coexistence Between 802.15.4 and 802.11: A Measurement-based Study," Cognitive Radio Oriented Wireless Networks and Communications, 2008. CrownCom 2008. 3rd International Conference on , vol., no., pp.1-6, 15-17 May 2008. doi: 10.1109/CROWNCOM.2008.4562460. URL: $\mathrm{http}$ ://ieeexplore.ieee.org/stamp/stamp.jsp?tp=\&arnumber=4562460\&isn umber $=4562434$

[2] Batteries in bulk webshops, e.g. http://www.batteryjunction.com, http://www.batteriesinaflash.com, etc.

[3] K. Casier, L. Van Halewyck, S. Verbrugge, D. Colle, M. Pickavet, P. Demeester, Extending operational models to perform micro optimizations, Proceedings of ECOC2007, the 33rd European Conference and Exhibition on Optical Communication, 16-20 September 2007, Berlin, Germany

[4] L. Tytgat M. Barrie, et all., "Techno-economical Viability of Cognitive Solutions for a Factory Scenario", 2011 IEEE International Symposium on Dynamic Spectrum Access Networks (DySPAN), pp. 254-264, 3-6 May 2011

[5] M. Barrie, L. Tytgat, et al., "Techno-Economic evaluation of Cognitive Radio in a Factory Scenario", in proceedings of Perfomance Evaluation of Cognitive Radio Networks (PE-CRN) 2011, 9-13 may 2011. 